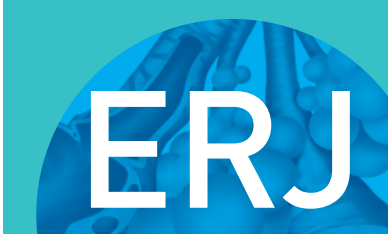

open research
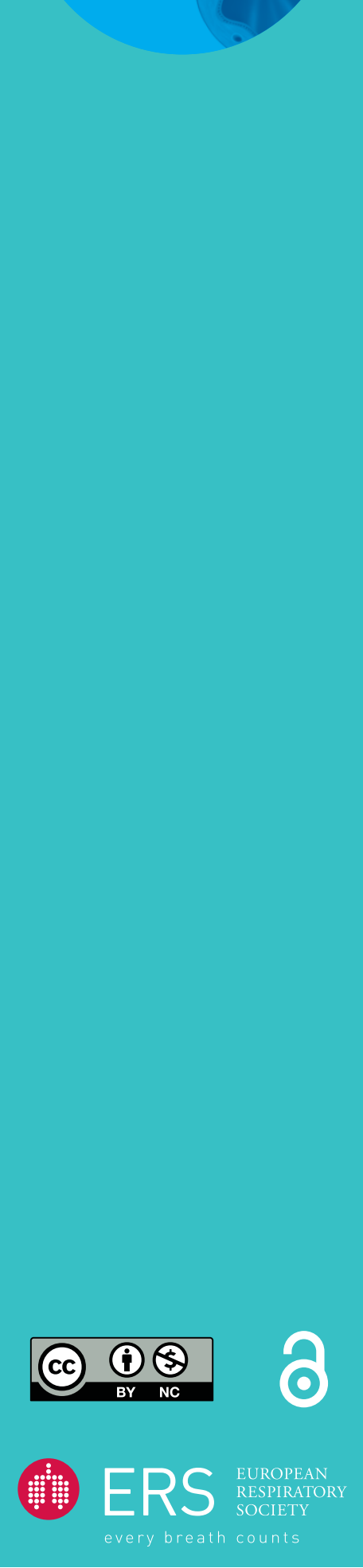

\section{Pregnancy after lung and heart-lung transplantation: a French multicentre retrospective study of 39 pregnancies}

\author{
Charlotte Bry ${ }^{1}$, Dominique Hubert ${ }^{2}{ }^{2}$, Martine Reynaud-Gaubert ${ }^{3}$, \\ Claire Dromer ${ }^{4}$, Hervé Mal ${ }^{5}$, Antoine Roux ${ }^{6}$, Véronique Boussaud ${ }^{7}$, \\ Johanna Claustre ${ }^{8}$, Jérôme Le Pavec ${ }^{9}$, Muriel Murris-Espin ${ }^{10}$ and \\ Isabelle Danner-Boucher ${ }^{1}$
}

Affiliations: ' Service de Pneumologie, CHU de Nantes, Nantes, France. ${ }^{2}$ Service de Pneumologie, Hôpital Cochin, Paris, France. ${ }^{3}$ Centre de Ressource et de Compétences de la Mucoviscidose Adulte, Equipe de Transplantation Pulmonaire, CHU Nord, Marseille, France. ${ }^{4}$ Service de Pneumologie, CHU de Bordeaux, Bordeaux, France. ${ }^{5}$ Service de Pneumologie, Hôpital Bichat-Claude Bernard, Paris, France. ${ }^{6}$ Service de Pneumologie, Hôpital Foch, Suresnes, France. ${ }^{7}$ Service de Pneumologie, Hôpital Européen Georges Pompidou, Paris, France. ${ }^{8}$ Clinique Universitaire de Pneumologie, Pôle Thorax et Vaisseaux, CHU de Grenoble, Grenoble, France. ${ }^{9}$ Service de Chirurgie Thoracique et Vasculaire, Centre Chirurgical Marie Lannelongue, Le Plessis Robinson, France. ${ }^{10} \mathrm{CRCM}$ Adulte, Service de Pneumologie-Allergologie, CHU de Toulouse, Toulouse, France.

Correspondence: Charlotte Bry, Service de Pneumologie, Hôpital G. et R. Laennec, Boulevard Jacques Monod, 44093 Nantes Cedex 1, France. E-mail: charlotte.bryachu-nantes.fr

ABSTRACT Pregnancy after lung and heart-lung transplantation remains rare. This French study deals with change in lung function after a pregnancy and the maternal and newborn outcomes.

We retrospectively included 39 pregnancies in 35 women aged $>20$ years. Data on patients, course of pregnancies and newborns were collected from nine transplantation centres.

Mean age at time of pregnancy was 28 years. Cystic fibrosis affected $71 \%$ of patients. Mean $\pm \mathrm{SD}$ time between transplantation and pregnancy was $63 \pm 44$ months. 26 births occurred (67\%) with a mean term of 36 weeks of amenorrhoea and a mean birthweight of $2409 \mathrm{~g}$. Prematurity was observed in 11 cases (43\%). Forced expiratory volume in $1 \mathrm{~s}$ was $83.9 \%$ of predicted before pregnancy and $77.3 \%$ of predicted 1 year after the end of pregnancy $(p=0.04) .10$ patients developed chronic lung allograft dysfunction after delivery. Nine patients died at a mean \pm SD time after transplantation of $8.2 \pm 7$ years and a mean \pm SD time after pregnancy of $4.6 \pm 6.5$ years.

These data show that pregnancy remains feasible in lung and heart-lung transplant recipients, with more frequent maternal and newborn complications than in the general population. Survival in this cohort appears to be similar to the global survival observed in lung transplant recipients. Planned pregnancy and multidisciplinary follow-up are crucial.

@ERSpublications

Pregnancy in lung and heart-lung recipients remains rare but possible. There is a significant decrease in $\mathrm{FEV}_{1}$ pre- and post-pregnancy, but overall outcomes are reassuring. Specialised, multidisciplinary follow-up is necessary. http://bit.ly/31iXxov

Cite this article as: Bry C, Hubert D, Reynaud-Gaubert $\mathrm{M}$, et al. Pregnancy after lung and heart-lung transplantation: a French multicentre retrospective study of 39 pregnancies. ERJ Open Res 2019; 5: 00254-2018 [https://doi.org/10.1183/23120541.00254-2018].

Received: 20 Dec 2018 | Accepted after revision: 29 July 2019

Copyright $\odot E R S$ 2019. This article is open access and distributed under the terms of the Creative Commons Attribution Non-Commercial Licence 4.0. 


\section{Introduction}

Lung transplantation is a valid treatment for selected patients with end-stage respiratory failure [1]. Advances in this field have made it possible to improve life expectancy and quality of life. More than $43 \%$ of lung transplant patients are women of childbearing age. At its best, controlling complications and ensuring a stable clinical condition has made it possible to allow women with solid organ transplants to undertake pregnancies. However, there are still ethical questions [2]. The largest studies available relate to women with renal transplants, and describe an increased risk of pregnancy-induced hypertension, pre-eclampsia, gestational diabetes and premature childbirth [3-5]. These studies were the main references used for the recommendations regarding pregnancy management in women with solid organ transplants [6]. As for women with lung transplantation, there are a limited number of studies, usually monocentric and involving small groups of patients [7-11]. According to the important National Transplantation Pregnancy Registry (NTPR) [12], a higher risk of complications (death, allograft rejection and premature childbirth) is found. Currently, there are no specific international recommendations regarding pregnancy management in women with lung transplantation; only expert recommendations are available [13].

The main objective of this multicentre study was to assess the impact of pregnancy on lung allograft function. Secondary objectives were to describe maternal complications and child health.

\section{Methods}

This French study was approved by the Nantes University Hospital Centre Ethics Committee (GNEDS) and data were reported to the Commission Nationale Informatique et Liberté (CNIL) (the French data protection authority). Written informed consent was obtained.

\section{Patients}

We retrospectively included all pregnancies beginning between January 1, 1991 and April 1, 2013 in women who underwent lung transplantation (single lung, bilateral lung or heart and lung) in France.

Recruitment was carried out by contacting the attending physicians of the 11 French lung transplantation centres in France. Data were retrieved from local medical records.

Pre-defined exclusion criteria were women beginning pregnancy aged $<18$ years and adult women who had a legal guardian or were wards of the court.

\section{Study design}

The main criterion utilised (judgement criteria) was forced expiratory volume in $1 \mathrm{~s}\left(\mathrm{FEV}_{1}\right)$ at 1 year after the end of pregnancy. We compared this value with the pre-pregnancy $\mathrm{FEV}_{1}$, defined as the last available value before pregnancy. A decrease of $\geqslant 5 \%$ in the absolute $\mathrm{FEV}_{1}$ value was considered significant [14]. For patients who received several transplants, we analysed the last transplant before pregnancy.

Baseline data were collected (age at time of transplant, age at start of pregnancy, underlying disease and surgical procedure). $\mathrm{FEV}_{1}$, body mass index (BMI), renal failure, diabetes, arterial hypertension, acute cellular rejection and chronic lung allograft dysfunction (CLAD) were assessed before pregnancy, at the end of pregnancy and 1 year after the end of pregnancy. CLAD was defined according to the current classification system [15].

Specific data on the pregnancy were also retrieved: prior consent, assisted or unassisted pregnancy, outcomes, immunosuppressive regime and infections. Characteristics related to the newborn (weight, initial health and breastfeeding) were reported. The newborn's health was considered as normal when the Apgar score (an evaluation of major vital functions at birth) at 5 min was 10.

\section{Statistical analyses}

Statistical analyses were mainly descriptive. The main criterion was the $\mathrm{FEV}_{1}$ at 1 year after the end of pregnancy. This value was compared with the pre-pregnancy $\mathrm{FEV}_{1}$ using a matched-pair t-test.

A p-value $<0.05$ was considered significant. Analyses were carried out with SPSS Statistics version 19 (IBM, Armonk, NY, USA) and SAS version 9.3 (SAS Institute, Cary, NC, USA).

\section{Results}

We included 35 patients with 39 monitored pregnancies in 11 centres in France. Two centres included 22 pregnancies and two others centres did not include any pregnancies. There were no excluded patients based on our pre-defined criteria. Seven pregnancies (18\%) required assistance with fertility treatment $(\mathrm{n}=33$ in vitro fertilisation, $\mathrm{n}=3$ ovarian stimulation with insemination and $\mathrm{n}=1$ missing data) and seven started without the approval of the transplant team. 


$\begin{array}{lc}\text { TABLE } 1 \text { Baseline patient characteristics } & 35 \\ \text { Patients } & 23 \pm 5 \\ \text { Age at transplant years } & 28 \pm 5 \\ \text { Age at start of pregnancy years } & 19.8 \pm 2.5 \\ \text { BMI before pregnancy } \mathbf{k g} \cdot \mathbf{m}^{-2} & 83 \pm 25 \\ \text { FEV }_{\mathbf{1}} \text { before pregnancy \% pred } & 63 \pm 44 \\ \text { Time between transplant and pregnancy months } & 25(71) \\ \text { Underlying disease } & 7(20) \\ \text { Cystic fibrosis } & 3(9) \\ \text { Pulmonary hypertension } & 22(63) \\ \text { Others } & 13(37) \\ \text { Type of surgery } & \text { Bilateral lung }\end{array}$

Data are presented as $\mathrm{n}$, mean \pm SD or $\mathrm{n}(\%)$. BMI: body mass index; FEV 1 : forced expiratory volume in $1 \mathrm{~s}$.

Mean \pm SD age was $28 \pm 5$ years at the beginning of pregnancy. The majority of women underwent bilateral lung transplant $(\mathrm{n}=22(63 \%))$, none underwent single lung transplant and in the residual $37 \%$ they had a concomitant heart transplant. One woman became pregnant after a second bilateral lung transplantation. The underlying diagnosis was cystic fibrosis for 25 patients (71\%). Mean \pm sD time between transplantation and the start of pregnancy was $63 \pm 44$ months (table 1 ).

26 pregnancies (67\%) resulted in childbirth. As for the 13 other pregnancies, there were seven spontaneous miscarriages, three therapeutic terminations of pregnancy, two voluntary interruptions of pregnancy and one miscarriage resulting from maternal death at 6 weeks of amenorrhoea (WA). For two patients, therapeutic termination of pregnancy was done due to an excessively premature pregnancy regarding transplant; for the third, therapeutic termination of pregnancy was done due to an unstable respiratory condition (table 2).

Complications during pregnancy included gestational diabetes in two, pregnancy-induced hypertension in three, pre-eclampsia in one, renal failure in one and eight infections including one severe acute pneumonitis resulting in death at $6 \mathrm{WA}$ (table 3). No acute cellular rejection or CLAD was diagnosed during pregnancy. Immunosuppressive treatment included a combination of corticosteroid $(n=24)$, cyclosporine $(n=17)$, tacrolimus $(n=16)$ and azathioprine $(n=22)$.

There were 26 newborns, $90 \%$ of which were in normal clinical condition at birth. Childbirth was induced for $50 \%$ of the births and $48 \%$ of the patients gave birth via caesarean section. Two newborns presented serious complications at birth as a result of extreme prematurity ( 22 and $28 \mathrm{WA}$ ). The mean $\pm \mathrm{SD}$ term was $36 \pm 5 \mathrm{WA}$, with $43 \%$ prematurity $(<37 \mathrm{WA})$. Among the 11 premature newborns, five resulted from spontaneous delivery (45\%), four from induced premature delivery (36\%) and two sets of data were missing. The mean \pm SD birthweight was $2409 \pm 920 \mathrm{~g}$ and $48 \%$ presented with low birthweight $(<2500 \mathrm{~g})$. $96 \%$ of the children were not breastfed. No malformation was reported (table 4).

The mean $\mathrm{FEV}_{1} \%$ pred before pregnancy was $82.5 \%$ and at 1 year after the end of pregnancy was $77.3 \%$ $(\mathrm{p}=0.04)$ (figure 1).

At 1 year after the end of pregnancy, we noted a decrease of $\mathrm{FEV}_{1} \%$ pred of $>5 \%$ in 14 pregnancies (13 patients, one of whom had two pregnancies). As for the other 23 pregnancies (21 patients, two of whom had two pregnancies each), respiratory functions did not change significantly. Two patients died before 1 year post-pregnancy. 11 patients experienced one or more acute cellular rejection episode. 15 patients

\section{TABLE 2 Pregnancy outcomes}

$\begin{array}{lc}\text { Spontaneous miscarriage } & 7(18) \\ \text { Voluntary termination of pregnancy } & 2(5) \\ \text { Therapeutic termination of pregnancy } & 3(8) \\ \text { Death of pregnant women } & 1(3) \\ \text { Birth } & 26(67)\end{array}$

Data are presented as n (\%). 


$\begin{array}{lc}\text { TABLE } 3 \text { Maternal complications during pregnancy } & \\ \text { Diabetes } & 19(51) \\ \text { Before pregnancy } & 18(55) \\ \text { During pregnancy } & 2(6) \\ \text { Gestational diabetes } & 18(51) \\ \text { Arterial hypertension } & 17(59) \\ \text { Before pregnancy } & 3(10) \\ \text { During pregnancy } & \\ \text { Pregnancy-induced hypertension } & 21(58) \\ \text { Renal failure } & 19(61) \\ \text { Before pregnancy } & 1(3) \\ \text { During pregnancy } & 14(39) \\ \text { New during pregnancy } & 0(0) \\ \text { Acute rejections } & 11(33) \\ \text { Before pregnancy } & 8(24) \\ \text { During pregnancy } & \\ \text { After pregnancy } & \\ \text { Infections during pregnancy } & \end{array}$

\section{TABLE 4 Newborn characteristics}

\section{Newborns \\ Pregnancy term WA \\ Induced labour \\ Caesarean section \\ Premature childbirth (<37 WA) \\ Weight at birth $\mathrm{g}$ \\ Underweight \\ Breastfeeding}

$9(51)$

$8(51)$

7 (59)

$1(58)$

$9(61)$

4 (39)

$0(0)$

$11(33)$

8 (24)

Data are presented as $n$, mean \pm SD or $n(\%)$. WA: weeks of amenorrhoea.

Overall population

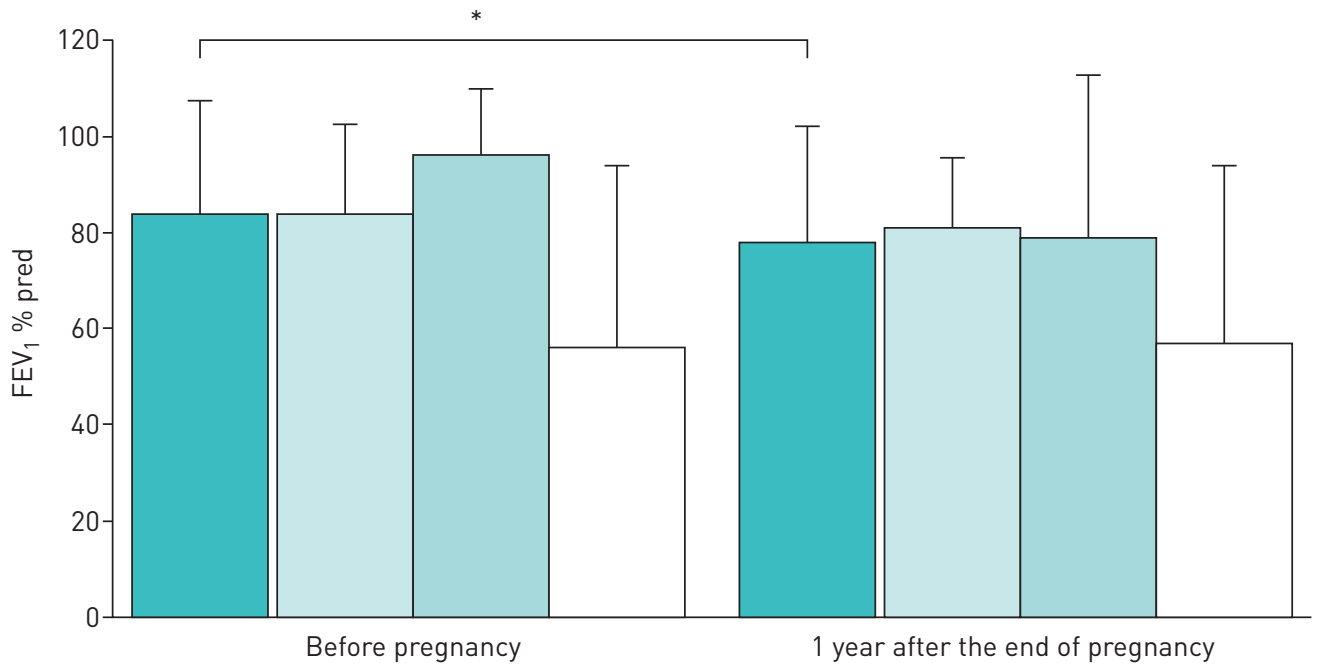

FIGURE 1 Evolution of forced expiratory volume in $1 \mathrm{~s}\left(\mathrm{FEV}_{1}\right)$ before pregnancy and 1 year after the end of pregnancy: overall population and subgroup analysis according to chronic lung allograft dysfunction (CLAD) status. Data are represented as mean \pm SD FEV $1 \%$ pred. *: $p<0.05$. 


\begin{tabular}{lc} 
TABLE 5 Characteristics of chronic lung allograft dysfunction (CLAD) patients & 15 \\
CLAD patients & $5(16)$ \\
CLAD diagnosis & $0(0)$ \\
$\quad$ Before pregnancy & $10(46)$ \\
During pregnancy & $22.5 \pm 14.6$ \\
After pregnancy & $78.1 \pm 38.2$ \\
Time of appearance of CLAD after pregnancy months (n=10) & $5(33)$ \\
Time between CLAD and transplant months (n=10) & $3(20)$ \\
Patient outcomes & $7(47)$ \\
Stable & \\
Retransplantation & \\
Death & \\
\hline Data are presented as $n$, mean $\pm S D$ or $n(\%)$. &
\end{tabular}

had CLAD, 10 of which were reported after pregnancy (one CLAD was reported after two pregnancies) (table 5). For patients with CLAD before pregnancy, $\mathrm{FEV}_{1}$ was stable 1 year before pregnancy; mean $\pm \mathrm{SD}$ $\mathrm{FEV}_{1} \%$ pred was $56.4 \pm 37.3 \%$ right before pregnancy and $57.6 \pm 36.8 \% 1$ year after the end of pregnancy $(\mathrm{p}=0.5)$. The median (interquartile range (IQR)) follow-up after transplantation was $12.5(8.0-18.5)$ years. At the end of follow-up, nine patients had died (one during pregnancy) within a mean $\pm \mathrm{SD}$ time of 4.6 \pm 6.5 years after pregnancy and $8.2 \pm 7.0$ years after transplantation. At 12 years, survival was $71.2 \pm 9.0 \%$ (figure 2). Seven of the deceased patients had CLAD. Five patients died of CLAD-related terminal respiratory failure, two of pulmonary infection, one of pulmonary embolism and one of heart failure.

\section{Discussion}

To the best of our knowledge, this study is the largest cohort of pregnancies in women with lung and heart-lung transplantation, and the first to report a significant deterioration of lung function 1 year after the end of pregnancy. We found a decrease of $>5 \%$ of $\mathrm{FEV}_{1} \%$ pred, which was not apparently related to an increase risk of CLAD or acute cellular rejection.

At present, around 100 pregnancies have been reported for women with lung or heart-lung transplantation. 35 are recorded in the US NTPR registry [6], which compiles pregnancies in women with solid organ transplants. There is no precise description of respiratory function changes, although they reported a greater number of graft rejections, compared with other organs, within 2 years after pregnancy. The single-centre study conducted by THAKRAR et al. [16] on 14 pregnancies found post-pregnancy $\mathrm{FEV}_{1}$ stability ( $1.1 \%$ variation), but it is noteworthy that they reported one patient who presented with severe and rapid bronchiolitis obliterans syndrome at the onset of pregnancy and was excluded from the lung function analysis.

FIGURE 2 Kaplan-Meier survival analysis. At 12 years, survival was $71.2 \pm 9.0 \%$. $n=35$ patients.

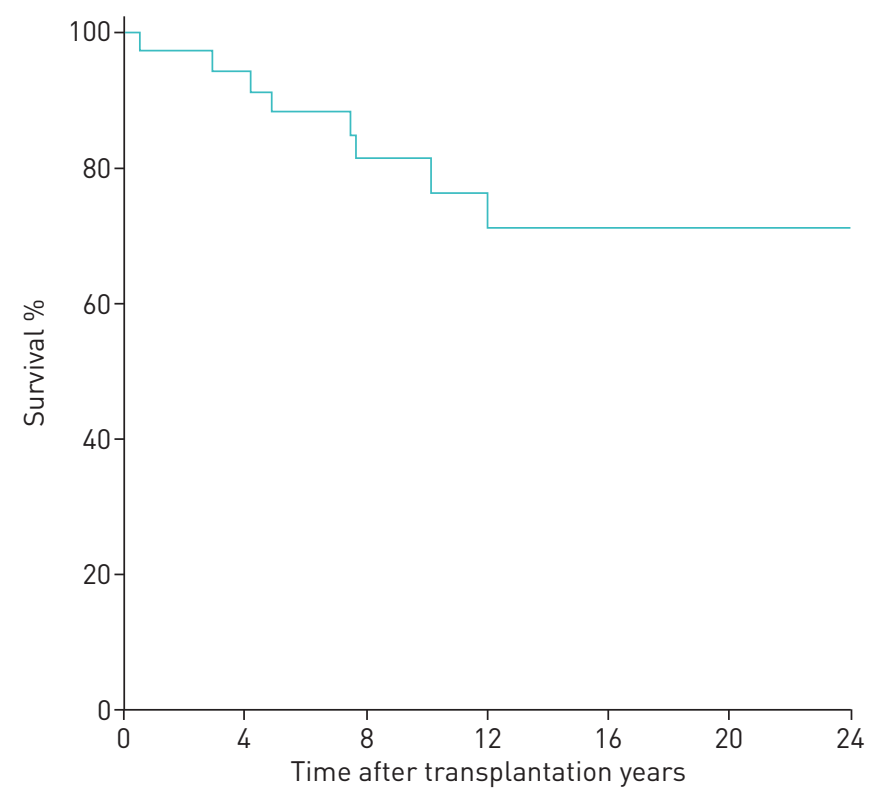


In our study, 14 patients had an absolute $\mathrm{FEV}_{1}$ decrease of $>5 \%$ and 10 had an absolute $\mathrm{FEV}_{1}$ decrease $>10 \% 1$ year after the end of pregnancy. Among these 14 patients, only six patients developed CLAD after pregnancy. As for the other eight patients, one had developed CLAD before being pregnant and worsened lung function during pregnancy, while no cause for $\mathrm{FEV}_{1} \%$ pred decrease was identified in the remaining seven. Among the latter, no diagnosis of CLAD was recorded at the 1 -year time-point. It is difficult to assert if this lung function decrease should be considered as clinically significant. In our study, the survival appeared reasonable. At 12 years, the survival was $71 \%$ and the estimated median survival was 24 years. Median survival conditional on survival to 1 year for adult lung transplants was 7.9 years and at 12 years after transplantation, $30 \%$ were alive [17].

It is important to note that our study population is not comparable in every way to other described cohorts. Cystic fibrosis affected $>70 \%$ of our patients compared with $57 \%$ in both SHANER et al. [12] and Thakrar et al. [16]. As a consequence, mean age was 28 years, which is younger than in previous studies $[12,16]$. In France, cystic fibrosis was the primary underlying disease for lung transplantation at the time of the study [18], which is not the case in the USA, for example, where chronic obstructive pulmonary disease/emphysema predominates. In contrast to other studies, no patient presented any new acute rejection or CLAD during pregnancy. SHANER et al. [12] reported a rate of $17 \%$ acute rejection. We can hypothesise that invasive tests such as transbronchial biopsies were not likely to be performed during pregnancy and thus acute rejection might have been underdiagnosed. Nonetheless, some authors describe possible immunotolerance mechanisms during pregnancy due to a decreased T-lymphocyte response [19]. In the post-partum period, we reported four acute rejections in two patients within the 6 months following childbirth. The first patient quickly developed CLAD. The second patient presented progressive aggravation of CLAD. 10 patients developed CLAD after pregnancy, with a mean time of 22.5 months after pregnancy and 6.5 years after the transplant. At present, three patients have been retransplanted and four have died. The time between transplant and post-pregnancy CLAD is hardly comparable to that normally found in people as a whole that have transplants. The prevailing trend is $30 \%$ at 3 years and $50 \%$ at 5 years [20]. In our study, the mean time between transplantation and pregnancy was 63 months. Pregnancy is contraindicated in patients with unstable health and presenting with serious, rapid post-transplant complications such as CLAD. This is an important bias that explains the longer timeframe. The post-partum period is considered at risk for graft rejection [21]. First, this can be due to the sudden disappearance of the placenta and the decrease in circulating progesterone levels. Additionally, there is a sudden modification of volume distribution, thus making it difficult to monitor the immunosuppressive treatment. Finally, there can be issues with treatment compliance due to post-partum depression [21], as was the case for one of our patients who developed CLAD at 36 months post-partum. In some centres, corticosteroid dose was increased for a few weeks after childbirth in order to account for this presumed risk.

The time period of 63 months between pregnancy and transplant concurs with the timeframe of at least 1 year recommended by the NTPR and the American Society of Transplantation for pregnancies after solid organ transplantation $[22,23]$. It is actually even higher, as in the previous studies $[12,16]$, due to more frequent complications following lung transplantation. The recent studies argue for a minimal 2-year timeframe between lung transplantation and pregnancy [12].

It is remarkable that $41 \%$ of the pregnancies were unplanned; the recent work by THAKRAR et al. [16] found a similar rate (36\%). Information on contraception is essential and should be provided promptly after transplantation. Before conception, patients should be informed of the possible maternal complications and the fetal/neonatal risks. The transplantation team, with the help of other specialists, should try to find the best time with the patient for pregnancy to ensure lung allograft stability and fetal wellbeing. None of the women in our study was on mycophenolate acid products during pregnancy. This antimetabolite is known to increase risks of miscarriage and congenital malformations $[24,25]$.

Assistance with fertility treatment was required for seven patients (18\%). Most of the women were aged $>30$ years and all but one of the patients had cystic fibrosis, a pathology known to affect fertility in both men and women [26]. Five of the medically assisted pregnancies (71\%) were successful. Medically assisted pregnancy can thus be a solution for these patients.

The birth rate in our study was $67 \%$, which was higher than in the literature $(42 \%$ in the study of THAKRAR et al. [16] and 56\% in SHANer et al. [12]). However, it is possible that not all premature miscarriages were reported.

Our results confirm data described previously, with a strong premature birth rate, a mean term of 36 WA and $48 \%$ premature births. $45 \%$ occurred because of spontaneous delivery. Mean birthweight connected with the premature births was $2409 \mathrm{~g}$. ARMENTi et al. [5] report a mean birthweight of $2285 \mathrm{~g}$. In the overall population, the mean birthweight is $3500 \mathrm{~g}$, which is significantly higher $(\mathrm{p}<0.001)$. 
These complications are described in patients with cystic fibrosis but without transplants [27]. Nonetheless, complications are also described in women with other organ transplantation, which raises the question of the role of immunosuppressive treatment. Additionally, there was no difference in premature birth rate and mean weight at birth between women with cystic fibrosis and the others.

Childbirth was often induced or via caesarean section. It is nonetheless clear that there were none but obstetric reasons for carrying out caesarean sections $[10,28]$. Childbirth was more complex and riskier than for other women due to the most frequent complications, such as pregnancy-induced hypertension with risk of pre-eclampsia, gestational diabetes and premature childbirth.

Only one child (4\%) was breastfed. Current data are unclear about breastfeeding management.

At present, no child seems to have psychomotor development problems or has presented with malformation.

Our study has several limitations. Although we report the largest cohort of pregnancies in lung and heart-lung transplantation, the number is limited.

It would be interesting to go back to the subgroup of patients presenting with a $\mathrm{FEV}_{1} \%$ pred decrease at 1 year after the end of pregnancy without any noted CLAD diagnosis and follow-up on their FEV 1 changes and mortality. These additional analyses might help to determine if the drop in $\mathrm{FEV}_{1}$ observed is clinically relevant and if pregnancy entails any actual risk to the lung allograft.

Data collection was retrospective. Moreover, obstetric data and child-related data were difficult to obtain, because these data are often not provided in the transplantation medical records. Some data, such as cytomegalovirus status or the presence of donor-specific antibodies, were not available for the earliest pregnancies reported in this study.

This study confirms the possibility of pregnancy in patients with lung and heart-lung transplantation. It also highlights the risks for their health and that of the newborn, and the need for clear information when a conception project is discussed. The pregnancy should be planned and carried out with a strong collaboration between the patient, the transplantation team and a specialised multidisciplinary medical team.

Acknowledgements: We thank the staff of all French lung transplantation centres for their help in data collection. We are furthermore grateful to Francois-Xavier Blanc and Adrien Tissot (CHU de Nantes, Nantes, France) for careful reading of the manuscript. We thank Béatrice Guyomarch (Institut du Thorax, CHU de Nantes, Nantes, France) for statistical analysis.

Conflict of interest: C. Bry has nothing to disclose. D. Hubert has nothing to disclose. M. Reynaud-Gaubert has nothing to disclose. C. Dromer has nothing to disclose. H. Mal reports nonfinancial support from Roche, Boehringer, CSL Behring and Novartis, outside the submitted work. A. Roux has nothing to disclose. V. Boussaud has nothing to disclose. J. Claustre has nothing to disclose. J. Le Pavec has nothing to disclose. M. Murris-Espin has nothing to disclose. I. Danner-Boucher has nothing to disclose.

\section{References}

1 Yusen RD, Christie JD, Edwards LB, et al. The Registry of the International Society for Heart and Lung Transplantation: thirtieth adult lung and heart-lung transplant report - 2013; focus theme: age. J Heart Lung Transplant 2013; 32: 965-978.

2 Ross LF. Ethical considerations related to pregnancy in transplant recipients. N Engl J Med 2006; 354: 1313-1316.

3 EBPG Expert Group on Renal Transplantation. European best practice guidelines for renal transplantation. Section IV: long-term management of the transplant recipient. IV.10. Pregnancy in renal transplant recipients. Nephrol Dial Transplant 2002; 17: Suppl. 4, 50-55.

4 McKay DB, Josephson MA. Pregnancy in recipients of solid organs - effects on mother and child. N Engl J Med 2006; 354: 1281-1293.

5 Armenti VT, Constantinescu S, Moritz MJ, et al. Pregnancy after transplantation. Transplant Rev 2008; 22: 223-240.

6 Coscia LA, Constantinescu S, Moritz MJ, et al. Report from the National Transplantation Pregnancy Registry (NTPR): outcomes of pregnancy after transplantation. Clin Transpl 2010; 7: 65-85.

7 Estensen M, Gude E, Ekmehag B, et al. Pregnancy in heart- and heart/lung recipients can be problematic. Scand Cardiovasc J 2011; 45: 349-353.

8 Parry D, Hextall A, Robinson VP, et al. Pregnancy following a single lung transplant. Thorax 1996; 51: 1162-1164.

9 Baron O, Hubaut J, Galetta D, et al. Pregnancy and heart-lung transplantation. J Heart Lung Transplant 2002; 21: 914-917.

10 Wu DW, Wilt J, Restaino S. Pregnancy after thoracic organ transplantation. Semin Perinatol 2007; 31: 354-362.

11 Zurbano F, López F, Fornet I, et al. Maternity and lung transplantation: cases in Spain. Arch Bronconeumol 2012; 48: 379-381.

12 Shaner J, Coscia LA, Constantinescu S, et al. Pregnancy after lung transplant. Prog Transplant 2012; 22: 134-140.

13 Vos R, Ruttens D, Verleden SE, et al. Pregnancy after heart and lung transplantation. Best Pract Res Clin Obstet Gynaecol 2014; 28: 1146-1162. 
14 Pennock BE, Rogers RM, McCaffree DR. Changes in measured spirometric indices. What is significant? Chest 1981; 80: 97-99.

15 Verleden GM, Raghu G, Meyer KC, et al. A new classification system for chronic lung allograft dysfunction. J Heart Lung Transplant 2014; 33: 127-133.

16 Thakrar MV, Morley K, Lordan JL, et al. Pregnancy after lung and heart-lung transplantation. J Heart Lung Transplant 2014; 33: 593-598.

17 Yusen RD, Edwards LB, Kucheryavaya AY, et al. The registry of the International Society for Heart and Lung Transplantation: thirty-first adult lung and heart-lung transplant report - 2014; focus theme: retransplantation. J Heart Lung Transplant 2014; 33: 1009-1024.

18 Agence de la Biomédicine. Greffe cardio-pulmonaire et pulmonaire. [Cardiopulmonary and pulmonary transplant.] 2103. www.agence-biomedecine.fr/annexes/bilan2013/donnees/organes/04-coeur-poumon/pdf/pcp.pdf Date last accessed: September 3, 2019.

19 Ma KK, Petroff MG, Coscia LA, et al. Complex chimerism: pregnancy after solid organ transplantation. Chimerism 2013; 4: 71-77.

20 Glanville AR, Aboyoun C, Klepetko W, et al. Three-year results of an investigator-driven multicenter, international, randomized open-label de novo trial to prevent BOS after lung transplantation. J Heart Lung Transplant 2015; 34: 16-25.

21 Mastrobattista JM, Gomez-Lobo V. Pregnancy after solid organ transplantation. Obstet Gynecol 2008; 112 919-932.

22 McKay DB, Josephson MA, Armenti VT, et al. Reproduction and transplantation: report on the AST Consensus Conference on Reproductive Issues and Transplantation. Am J Transplant 2005; 5: 1592-1599.

23 Deshpande NA, Coscia LA, Gomez-Lobo V, et al. Pregnancy after solid organ transplantation: a guide for obstetric management. Rev Obstet Gynecol 2013; 6: 116-125.

24 Kim M, Rostas S, Gabardi S. Mycophenolate fetal toxicity and risk evaluation and mitigation strategies. Am J Transplant 2013; 13: 1383-1389.

25 Sifontis NM, Coscia LA, Constantinescu S, et al. Pregnancy outcomes in solid organ transplant recipients with exposure to mycophenolate mofetil or sirolimus. Transplantation 2006; 82: 1698-1702.

26 Kopito LE, Kosasky HJ, Shwachman H. Water and electrolytes in cervical mucus from patients with cystic fibrosis. Fertil Steril 1973; 24: 512-516.

27 Lau EMT, Barnes DJ, Moriarty C, et al. Pregnancy outcomes in the current era of cystic fibrosis care: a 15-year experience. Aust NZ J Obstet Gynaecol 2011; 51: 220-224.

28 Troché V, Ville Y, Fernandez H. Pregnancy after heart or heart-lung transplantation: a series of 10 pregnancies. $\mathrm{Br}$ J Obstet Gynaecol 1998; 105: 454-458. 